\title{
LA INCLUSIÓN EDUCATIVA: UNA TAREA QUE LE COMPETE A TODA UNA SOCIEDAD
}

\section{M.Sc. Ronald Soto Calderón'}

Resumen: Este artículo se escribe con referencia a las ideas acerca del proceso de integración e inclusión de personas con necesidades educativas especiales al aula. Se señala cómo la sociedad debe involucrarse para hacer de la integración una realidad, y cómo estas experiencias deben posibilitar el que haya estudiantes con NEE incluidos en clases regulares.

También, con el fin de construir un proceso de inclusión en ambientes institucionales, este artículo recoge aspectos que deben ser imperativos de acción para las instituciones educativas, sean de primaria o secundaria, y de este modo, ofrecerles a todos los estudiantes, con o sin discapacidad (necesidades educativas especiales), la posibilidad de involucrarse en programas educacionales, y ayudarles a ser parte de la sociedad en diferentes formas: como estudiante, trabajador o miembro de la familia en la cual viven.

En otras palabras, este artículo se escribe con la idea de hacer conciencia social sobre el papel que la institución educativa tiene, sobre todo, en cuanto a proporcionar oportunidades equitativas de progreso a todos los miembros de la sociedad; en un marco de respeto hacia las diferencias.

\begin{abstract}
This paper is written in reference to the ideas about the process of the Integration and Inclusion of persons with special education needs, it refers to the ways in which the Society must participate in order to make the Integration a reality, and how this experiences must transform the possibility of having students included in regular classes.

Also, in order to create a real Inclusion process of the person in the educational environments, the paper refers to the aspects the institutions must do, to offer the students with or without disabilities the possibilities to be come involved in educational programs, and help them to be part of the society in many ways, that means as a student, worker or part of the family in which they live.

In other words, the paper is written with the idea of make conscientious in the society, about the role that it makes take, in order to give opportunities to all their members with or without disabilities to be each time better and respectful of the differences and diversity, also, given them the possibility to participate in equality and comparing opportunities.
\end{abstract}

Palabras claves: Integración/Integration, Inclusión/Inclusion, Necesidades educativas especiales /Special Educational Needs.

\section{A manera de introducción}

Mucho se ha oído hablar de respuesta a las necesidades educativas de los estudiantes que han sido trasladados de servicios de apoyo fijo o de escuelas de educación especial a escuelas regulares y, a menudo, se ha hecho referencia a los conceptos de integración, participación y adecuación curricular; en fin, de todo un sinnúmero de términos que podrían o no dar sentido a los procesos que se desarrollan en este ámbito, tanto en el país como en el marco mundial.

\footnotetext{
${ }^{1}$ Magister en Educación Especial -ICSE- Universidad Pontificia de Comillas, Madrid, España; Magister en Evaluación Educativa de la Universidad de Costa Rica; Sub director y profesor de la Escuela de Orientación y Educación Especial de la Universidad de Costa Rica; Profesor de la División de Educación Básica del Centro de Investigación y Docencia en Educación de la Universidad Nacional. Profesor de la Maestría en Estudios Interdisciplinarios sobre Discapacidad; Investigador del Instituto de Investigación para el Meioramiento de la Educación Costarricense (IIMEC). e-mail: ronaldsc@cariari.ucr.ac.cl
}

Artículo recibido: 26 de agosto, 2003

Aprobado: 24 de noviembre, 2003 
Algunas veces, pareciera ser que los docentes asumen su tarea más por una cuestión personal que por una profesional, y que el principio del derecho a la educación, lejos de legitimar que todos los miembros de una sociedad participen en igualdad de condiciones y equiparación de oportunidades (Asamblea Legislativa, 1996) sin importar su condición, favorezca más bien una situación en la cual nadie tiene claro lo que se hace en las aulas a las que asisten los estudiantes con necesidades educativas especiales.

En los últimos años, y como producto de los procesos de globalización y los cambios en los enfoques de la educación especial, los medios educativos han empezado a hablar de "inclusión educativa", término que de alguna manera pretende justificar la falta de claridad en cuanto a lo que se propone desde el Sistema Educativo Nacional con respecto a la unificación de un currículo propuesto por las altas jerarquías.

No se puede hablar de inclusión en tanto no se haya realizado un análisis y discusión de los diferentes procesos en que se ha enmarcado la experiencia educativa de las personas con necesidades educativas especiales; así como los procesos de formación de los docentes y otros profesionales relacionados con estas personas.

La historia ha demostrado que no solo es necesario un cambio en el uso de los términos; asimismo, es claro que lo que funciona en una situación o país, no necesariamente debe convertirse en moda o ser asumido por otra nación, ni tampoco debe verse como verdad absoluta. Hoy en día, los cambios que se han generado a partir de la experiencia del ser humano, la cotidianidad y las formas de abordar las realidades estudiadas, obligan a replantear las formas de trabajo, las experiencias y todo aquello que implique un abordaje integral del quehacer profesional docente, en el cual, el entorno se constituye en elemento primordial.

Por lo tanto, cuando se habla de inclusión, se crean expectativas para todas las personas y grupos que tienen que ver, en su trabajo, con personas que requieren ciertos apoyos para enfrentar no solo su interacción y aprendizaje en el aula, sino también en su familia y comunidad. Es decir, se debe tomar en cuenta todo aspecto relacionado con la cultura en la que se desarrollan las personas; en este sentido, comenta Heward (1997) "(...) la herencia cultural que un niño recibe también varía enormemente. No podemos olvidar que el grupo cultural al que pertenecen los alumnos influye sobre sus valores y conductas" ( $p$. 
62), y por lo tanto, estos aspectos deben ser considerados en el trabajo que se realice con los estudiantes.

Al hablar de inclusión se habla tolerancia, respeto y solidaridad, pero sobre todo, de aceptación de las personas, independientemente de sus condiciones. Sin hacer diferencias, sin sobreproteger ni rechazar al otro por sus características, necesidades, intereses y potencialidades, y mucho menos, por sus limitaciones; como anota Heward (1997) “ (...) para sobrevivir, un grupo social debe adaptar y modificar el ambiente en el que vive" (p. 62).

\section{Aspectos generales de la evolución de la Educación Especial: de la segregación a la inclusión}

La Educación Especial ha cambiado su panorama y por lo tanto, cambia también su forma de favorecer los procesos educativos de las personas con necesidades educativas especiales, pasando de desarrollar procesos asistenciales a procesos en los cuales se respeta la individualidad de las personas, en función de sus necesidades, características e intereses, y se pone énfasis en el entorno, como elemento que favorece o retrasa los procesos de participación de las personas con necesidades educativas especiales.

La educación especial por lo tanto, debe reconceptualizarse a la luz de los procesos de integración, y no se debe concebir al estudiante con necesidades educativas especiales como aquel que tiene una característica individual o un déficit que le es propio, sino más bien, se debe tomar en cuenta la participación del entorno, las políticas gubernamentales, los aspectos sociales y educativos, que facilitan que las dificultades que la persona experimenta en su desarrollo socioeducativo y emocional continúen obstaculizando su desarrollo.

Según Lou y López (2000) citando a Zabalza quien comenta que la integración escolar ha pasado por una serie de etapas, entre las cuales mencionan:

1. Reconocimiento del derecho a la educación de todos, sin embargo esto no reconoce que las personas con necesidades educativas especiales son "normales"

2. En relación con la anterior, la respuesta que se ha dado a estas personas es marginal y segregadora, por esta razón se han desarrollado servicios diferenciados en instituciones educativas,

3. Aparecer posteriormente la integración parcial 
Esta situación hace que la necesidad educativa especial se perciba desde una "óptica individualista" (Fulcher, citado por Echeita, Duk y Blanco (1995)); y por lo tanto se requiere que los estudiantes bajo esta condición sean atendidos en diferentes servicios, por diferentes profesores y en ambientes diferentes. Esto implicaría que los docentes que atienden esta población deban tener formación en los diversos aspectos necesarios para dar respuesta a las necesidades y características de la población con necesidades educativas especiales. Esta situación por otro lado, según Echeita y Martín, citados por Echeita y otros (1995) “(...) la respuesta educativa resultante es con frecuencia tan limitada que subestima la importancia de la interacción social como facilitadora del aprendizaje" (p.54).

Los aspectos antes mencionados llevan por lo tanto, a una propuesta que se base en el respeto a los derechos humanos y según la propuesta de Nirje y Nikkelsen se debe tomar en cuenta el principio de normalización y la filosofía de la integración, según Ortiz (2000) este principio hace referencia a que "(...) todo el mundo tiene derecho a utilizar los servicios normales de la comunidad y llevar una vida lo más normalizada posible" (p.6).

Tomando en cuenta principio de normalización, se ha plasmado el desarrollo de los servicios de atención educativa a las personas con necesidades educativas especiales, particularmente en Europa durante las décadas de los 70's y 80's se desarrolló lo conocido como integración escolar, de esta manera, en España en el año 1978 se introduce el principio de normalización, y se asume desde los principios teóricos de la integración escolar, la sectorización de los servicios educativos e individualizados de la enseñanza (Brown y Smith, 1996; Ortiz, 2000), no obstante, es hasta el “(...) año 1995 cuando se aprueba el Real Decreto regulador del Programa de Integración Escolar del Ministerio de Educación y Ciencia, y cuyas características fueron: la implantación progresiva, la aplicación prudente, la dotación de recursos humanos y materiales y la evaluación del programa” (Ortiz, 2000, p.6).

La misma autora comenta que es en este año 1990 cuando se ha desarrollado la integración escolar dentro del sistema educativo general, pero anteriormente hubo todo un proceso de concienciación y cambio de mentalidad con respecto a las personas con necesidades educativas especiales.

De esta manera se puede decir que la escuela integradora lo que hace es romper barreras, quita paredes, busca las adecuaciones tanto de acceso como curriculares que 
permitan darle apoyo a las personas con necesidades educativas especiales en los procesos de acceso al currículo que le proponen en el Centro Educativo.

Los procesos que se desarrollan en España cobran especial importancia por el hecho de que a finales de los 70's aparece el informe Warnock en el cual se varía el concepto de personas con problemas de aprendizaje o dificultades en el área educativa, y aporta entonces el concepto de necesidades educativas especiales, con el cual se hace referencia a

“(...) un término que implica relatividad, interactividad, transitoriedad, y que remite a la interacción con el contexto tanto en la génesis como en la resolución de los conflictos, de forma que cualquier alumno pueda precisar de forma transitoria o permanente algún tipo de ayuda para proseguir un desarrollo académico y social normalizado, un currículo ordinario" (Ortiz, 2000, p.6).

Como se puede ver, el concepto encierra a diferentes colectivos que por diferentes características o condiciones pudieran requerir en un momento determinado de cierto tipo de apoyos, ayudas, adecuaciones y recursos, y esto se incluyó en la Convención de los Derechos del Niño hacia el Siglo XXI. Todo esto retomando también las ideas expresadas en la Declaración de Salamanca en 1994, y donde se pone énfasis a la educación de las personas con necesidades educativas especiales, en esta declaración también se incluyen aspectos relacionados con los procesos de inclusión, los cuales deberían ser abordados en las políticas educativas de los países que firmaron en la Conferencia de Salamanca.

Es importante ver como aún cuando se le da especial importancia a la atención de las personas con necesidades educativas especiales, esta se favorece al dar una respuesta general a la situación y favorece, asimismo, los procesos de solución de problemas, lo cual facilitará el acceso a los procesos de aprendizaje de todos los estudiantes y a la vez permitirá el desarrollo profesional de los docentes.

Es interesante ver como en Costa Rica hacia mediados de la década de los 70's se inician los primeros pasos en los procesos de integración, es así como se abren las aulas diferenciadas en las escuelas regulares, donde se atienden a personas con retraso mental leve y moderado, sin embargo en zonas donde no existen servicios de escuelas de educación especial, estos servicios de aulas diferenciadas podían asumir compromisos más severos. Por otro lado, se dan servicios para atender a los estudiantes que tienen dificultades de aprendizaje, en servicios de aula recursos, tanto en problemas de aprendizaje 
como de problemas de lenguaje y trastornos emocionales, en estos servicios el estudiante asiste regularmente al curso que le corresponde, y durante algunas horas de la semana recibe un servicio en el aula recurso.

Posteriormente, cuando los enfoques de la educación especial varían, los servicios que se les brinda a las personas con necesidades educativas especiales van cambiando de nombre, es así como las aulas diferenciadas se denominan posteriormente como aulas integradas. En este sentido y haciendo referencia a los últimos años, a partir de la aprobación de la Ley 7600: Ley de Igualdad de Oportunidades para las personas con Discapacidad y con la Política Educativa hacia el Siglo XXI, se abre la oportunidad de que los estudiantes con necesidades educativas especiales accedan al Sistema Regula de Educación, utilizando los mismos planes de estudio de todos los estudiantes, desarrollándose así la participación de los estudiantes en un ambiente educativo integrado y socializado, aspectos importantes en el proceso de integración.

Bajo estas condiciones se da la oportunidad a que las escuelas permitan el acceso a los procesos educativos de las personas con necesidades educativas especiales, sin distingo de condición, y fundamentando una práctica educativa que permita dar atención a las necesidades, características e intereses de todas las personas. Lo anterior con la idea de desarrollar una escuela en la que puedan participar en igualdad y equiparación de oportunidades todas las personas que estando en edad escolar deban participar de ella.

\section{La Inclusión: jentre el ser y el deber ser!}

La inclusión debe verse como una interacción que se genera en el respeto hacia las diferencias individuales y las condiciones de participación desde una perspectiva de igualdad y equiparación de oportunidades sociales, cualesquiera que sean los valores culturales, la raza, el sexo, la edad y "la condición" de la persona o grupo de personas. O sea, es necesario, en una sociedad como la costarricense, llevar a cabo procesos de concienciación que lleven a comprender quiénes somos y con quiénes compartimos; se debe identificar y tratar a las personas tal cual son "ellas mismas", "una de ellas", "el hijo de ...", y además, asegurar que cada individuo comprenda que siempre hay alguien que la escucha y la entiende; no necesariamente que le enseñe, pero sí que le comprenda. 
En este sentido, según anota la UNESCO (1994):

(...) La tendencia de la política social durante las dos últimas décadas pasadas ha sido fomentar la integración y la participación y luchar contra la exclusión. La integración y la participación forman parte esencial de la dignidad humana y el disfrute y ejercicio de los derechos humanos. En el campo de la educación, esta situación se refleja en el desarrollo de estrategias que posibiliten una auténtica igualdad de oportunidades ( $p$. 11).

Desde este punto de vista, es importante mencionar que las condiciones que determinan la desigualdad de la existencia de una persona en una sociedad concreta, son legitimadas por contextos históricamente construidos, y que por lo tanto, pueden ser revisados a partir de la participación ciudadana.

Con relación a las ideas anteriores, la UNESCO (1994) comenta:

La experiencia de muchos países demuestra que la integración de los niños y jóvenes con necesidades educativas especiales se consigue de forma eficaz en escuelas integradoras para todos los niños de la comunidad. Es en este contexto en el que los que tienen necesidades educativas especiales pueden avanzar en el terreno educativo y en el de la integración social. Las escuelas integradoras representan un marco favorable para lograr la igualdad de oportunidades y la completa participación, pero para que tengan éxito es necesario realzar un esfuerzo común, no solo de toda la escuela, sino también de los compañeros, padres, familias y voluntarios (p.11).

La inclusión debe ser concebida, además, como una organización política de la sociedad civil en la lucha por la inclusión de los colectivos minoritarios, cuya vía de acceso más importante es el acceso a la educación, aunque no la única. Por lo tanto, la integración educativa y escolar están relacionadas con la inclusión a la educación básica regular de todas las personas independientemente de sus condiciones; de esta manera, la decisión de si las personas se ven involucrados en procesos de intervención o acción correctiva, o participan de un modelo educativo, corresponde a la familia y la sociedad a la cual pertenecen, y no es tarea de los expertos, sean estos técnicos, científicos o profesionales, como se ha querido asumir.

Se puede decir, entonces, que se está ante un hecho social y no natural. Se trata más de una construcción social que de una construcción que se desarrolla sobre las recomendaciones planteadas en la Declaración de Salamanca (UNESCO, 1992), va más allá, ya que está relacionada con los Derechos Humanos, con los Derechos de las niñas y los niños, de las Normas Uniformes sobre la Igualdad de Oportunidades para las Personas con Discapacidad, con la Declaración Mundial sobre Educación para Todos, y en fin, con toda aquella jurisprudencia que vela por el bien de todos los miembros de una sociedad. 
Teniendo en cuenta lo anterior, la inclusión educativa trasciende el ámbito de la escuela, y propone el derecho de todos al aprendizaje, y la atención a cada una de las personas según sus necesidades, características, intereses y potencialidades, cualquiera que sean sus características individuales.

Mediante la inclusión, se persigue brindar a las personas con alguna condición especial (permanente o transitoria), las mismas oportunidades, en igualdad y equiparación de oportunidades, que tienen todos los miembros de un país para acceder al proceso educativo; pero aquí no acaba el proceso. Es necesario que la persona pueda conectarse con redes interpersonales que traspasen todas las antiguas fronteras; y en este sentido, es importante contar con dos iniciativas desde la inclusión:

1. Lo que los estudiantes aprenden debe ser funcional para ellos en el entorno de una comunidad inclusiva (opuesto a lo que es solo funcional o cómodo en un entorno segregado).

Cuando se habla de currículo funcional se hace mención a la enseñanza de destrezas utilizadas en la vida diaria, que le permitan a la persona salir adelante en el mundo en que interactúa, de la forma más independiente que le sea posible.

2. Asegurarse de que el estudiante socialice o forme parte de una comunidad natural.

Por medio de la inclusión, se aprovecha cada experiencia, situación o evento que se da en un proceso interactivo, como una acción para el aprendizaje y para brindar todos los apoyos que las personas necesitan, teniendo en cuenta que el estudiante debe tener apoyos desde los aspectos económicos, hasta apoyos que le permitan su interacción con otras personas de su entorno, sean estas de carácter social o educativo, y siempre teniendo en cuenta sus necesidades, características, intereses y potencialidades, de tal manera que todos los apoyos converjan en una respuesta educativa que le permita el desarrollo integral al estudiante, sin importar sus condiciones. Lo anterior puede ejemplificarse en el siguiente diagrama: 


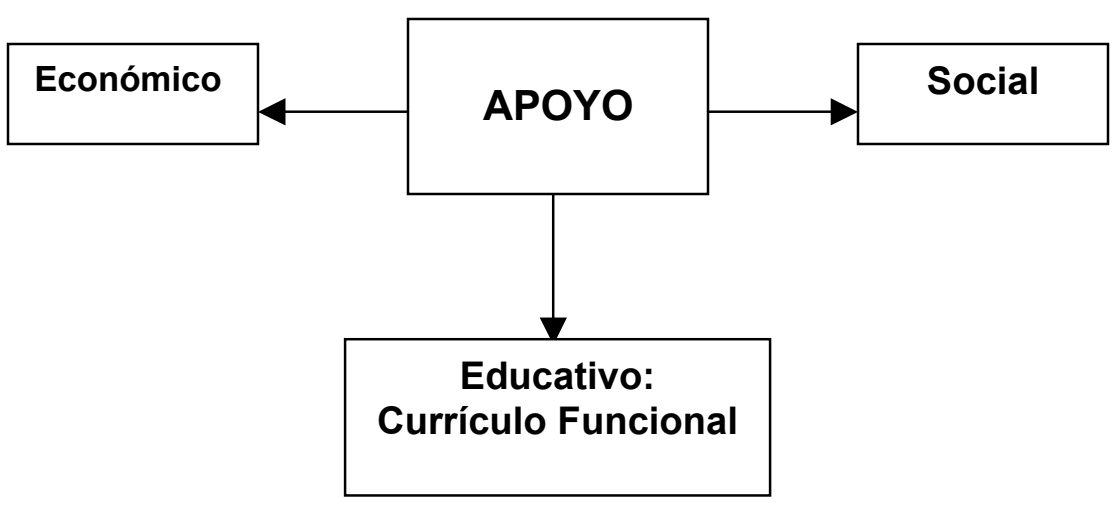

En este sentido, es importante acotar que sin la inclusión recíproca básica de la persona con alguna condición discapacitante, y sus compañeros primarios, en espacios diádicos de unión afectivo social, no pueden surgir formas más avanzadas de unión humana (cognitivas, comunicativas y lingüísticas) en las que tengan lugar los procesos naturales de aprendizaje y desarrollo de las personas. De esta manera, los individuos con NEE precisan ser incluidos en la co-cimentación (cimentación compartida) de sus propios espacios: cognitivo, comunicativos y lingüísticos.

Todas las personas con necesidades educativas especiales precisan tomar parte activa en situaciones, acontecimientos y experiencias que nutran la co-construcción (lo que se persigue es tener en cuenta los significados compartidos) de sus temas de aprendizaje y conversación.

Por lo anterior, es importante prestar atención a los cambios que se deben hacer en el centro educativo, con el fin de adaptar el proceso de enseñanza a todo el alumnado, o de lo contrario, los esfuerzos puestos individualmente en la integración de los estudiantes, pueden resultar, a la postre, estériles.

Lo anterior está íntimamente unido a la comprensividad del Sistema Educativo: es una opción de la política educativa, más allá de opciones personales a favor de promover al máximo el desarrollo de las personas con necesidades educativas especiales. Por lo tanto, al hablar de una educación inclusiva (o de una educación para todos), se debe prestar especial interés a la forma en que los estudiantes son incluidos en sus grupos, los apoyos que se les brindan y la forma en que estos les permiten acceder al proceso de enseñanza y aprendizaje, en función de sus necesidades, características, potencialidades, intereses y limitaciones. 
De alguna manera, la educación inclusiva hace referencia al derecho que todas las personas tienen a la educación (Asamblea Legislativa, 1996); o sea, es una construcción teórica y filosófica. Para operacionalizar esa definición conceptual se tiene que recurrir a su dimensión práctica. La dimensión práctica variará de contexto en contexto. Así, la forma real que tome la educación inclusiva, dependerá de los recursos humanos, del estado de desarrollo del sistema educativo relacionado con la formación pedagógica, de las facilidades físicas, de los recursos económicos, entre otros; este concepto de educación ha sido debatido y se ha relacionado con la dignidad y los derechos humanos.

Para fundamentar la integración de una manera más clara que camine a la inclusión, se debe pensar en que es la opción que existe entre la educación regular y especial, y no corresponde únicamente con un diagnóstico de un profesional o un experto, sino de la elección de un padre de familia bien orientado. De esta manera, para poder comprender y teorizar sobre educación especial, se debe llevar a cabo un proceso de teorización de la educación regular. Se trata de un enfoque incluyente de la educación regular y de la vida participativa de la sociedad, por parte de todas las personas sin ninguna distinción.

En este sentido, se dice que los países que han venido desarrollando los procesos de integración de manera acertada, parecen tener un potencial para la Educación Inclusiva, pero, ¿cuál sería la forma adecuada de llevar a cabo este proceso? Este modelo debe surgir de una revisión profunda de los esquemas sociales y educativos, y ser asumida por consenso social; de esta manera, es posible que se tenga éxito.

Cuando se habla de consenso, se hace referencia a un análisis de las políticas, en el cual, deben implicarse personas que tienen experiencia, y donde deben participar tanto los educadores de aula regular comprometidos con los cambios, como los partidarios de una educación especializada, ya que es importante que ellos debatan sobre la escuela. Además, se requiere una unificación conceptual y práctica sobre la Educación Inclusiva. Esto recalca lo mencionado anteriormente sobre la construcción teórica de la inclusión, la cual debe adquirir un significado que se ajuste al concepto en que se desarrolla, relacionándose con las finanzas, la capacidad y preparación de los docentes, el trabajo de un equipo nacional y lo que es capaz de ofrecer la educación regular, entre otras cosas.

\section{¿Cómo obtener los resultados más satisfactorios?}

Para lograr éxito en la inclusión se deben tener en cuenta dos aspectos, a saber: 
1. Trabajar con un equipo educativo que tenga la misma filosofía, que sea entusiasta y dispuesto a colaborar en un frecuente estudio (análisis, reestructuración, organización, entre otros) de los planes y los proyectos del centro.

2. Los grupos de amigos que se reúnen alrededor de una persona con necesidades educativas especiales, para apoyar su inclusión a la comunidad natural.

No obstante, no debe olvidarse que la integración es un proceso que ha perseguido que las escuelas sean más permeables a las necesidades de todos los estudiantes, y que por lo tanto, pudieran atender a aquellos que presentaban necesidades especiales y que hasta entonces eran sistemáticamente marginados de los centros ordinarios.

\section{Dificultades que se enfrentan en los procesos de inclusión}

Muchas son las dificultades que pueden presentarse en la puesta en práctica de los procesos de inclusión, entre ellos se pueden mencionar los siguientes:

1.Desde la formación de los profesionales y la cultura organizacional de los centros, hasta la falta de modelos (organizativos, curriculares y metodológicos) y el compromiso de la Administración Educativa en estos procesos. En este sentido según comenta Ramón-Laca (1998) “ (...) una de las críticas -al menos en Españaque se ha hecho a la integración escolar es que se ha implantado demasiado pronto, cuando el profesorado del sistema general no estaba preparado para recibir en sus aulas a alumnos con discapacidad" (p. 13).

2.Desconocimiento de la mayor parte de los miembros de los Centros en cuanto al enfoque de Inclusión (escuela para todos).

No se puede obviar que se han dado experiencias con el uso de material de UNESCO (Necesidades Especiales en el Aula) que han demostrado la forma en que se pueden dar pasos adelante, si el Centro se muestra favorable ante la Inclusión, y si viven el reto como una posibilidad de desarrollo personal y profesional.

Sin embargo, más que discutirse el concepto de Inclusión, es importante evaluar las distintas sociedades en las que se concretizan las acciones institucionales, examinando el contexto en el que ocurre la toma de decisiones y los intereses que las determinan.

Por lo tanto, las ideas no pueden ser entendidas si se aíslan de su contexto, sino más bien, como producto del análisis de los hombres frente a las condiciones sociales, políticas, económicas y regionales concretas de una sociedad. 
Es necesario, entonces, llevar a cabo un análisis de la diversidad que se atiende en las aulas, ya que es allí donde se deben concretar los principios democráticos de nuestra educación.

\section{¿En que áreas deben poner atención las escuelas inclusivas?}

Las condiciones para una escuela inclusiva son variadas y todo va a depender del contexto y realidad que viva cada centro educativo, ya que es ahí donde nacen los procesos de educación para todos.

Un primer momento implica una reflexión del profesorado sobre su práctica diaria y búsqueda de alternativas para mejorarla, tomando así conciencia de los principios fundamentales que orientan el trabajo de aula.

Entre los aspectos que deben considerarse, está el hecho de que todos los docentes son necesarios para lograr el éxito, y que el aprendizaje tiene un origen social, por lo que el aula debe ser abordada como comunidad educativa.

Partiendo de estos principios, se recomienda:

1.- Trabajo colaborativo entre los profesores: Trabajo en equipo que implica el planeamiento, las actividades y el desarrollo de la autoestima, entre otros. De alguna manera, implica crear conciencia de que todos los docentes participan de un proyecto, el cual está definido desde el centro.

2.- Estrategias de enseñanza y aprendizaje: Deben permitir la atención de todos los estudiantes que comparten la comunidad educativa, desarrollando el mismo plan de trabajo, pero contando además con todos los apoyos que requiere cada persona para participar en igualdad y equiparación de oportunidades, fomentando el trabajo colaborativo y cooperativo entre los estudiantes. Es importante que los docentes puedan dar un uso óptimo a los materiales y recursos con que cuentan él y la institución para llevar a cabo la práctica docente. Al respecto menciona Ramón-Laca (1998)

(...) al tutor le será tanto más fácil diseñar estrategias globales, cuanto mayor sea el conocimiento individual de sus alumnos, para lo que el profesor de apoyo es fundamental. La forma de incorporarse al proyecto global es peculiar y propia de cada alumno y de su propio nivel (p. 11).

3.- Atención a la diversidad desde el currículo: Es importante, en este aspecto, que los docentes logren procesos de capacitación que les permitan plantearse objetivos compartidos por todos los docentes del centro, donde se plasme la política tanto 
gubernamental como institucional, y que se aborden todos los aspectos relacionados con el proceso de enseñanza y aprendizaje, incluyendo los relacionados con la evaluación y seguimiento, áreas en las que muchos centros están ayunos de conocimiento e información. En este sentido, según anota Ramón-Laca (1998)

(...) Podríamos decir, por tanto, que el centro ordinario es hoy un lugar natural para escolarizar a un niño, a cualquier niño, con necesidades educativas especiales, o sin ellas. Pero debemos añadir "si da respuesta adecuada a ese niño", si cuenta con los recursos humanos y materiales suficientes para hacer frente a las necesidades del alumno y de su familia (p. 4).

4.- Administración y organización interna: Los centros educativos que participen en un proceso de inclusión o de escuela para todos, deben abocarse a acciones de análisis de centro, de estudios que permitan una autoevaluación y evaluación interna, y de conocimiento de la cultura organizacional; además, se debe contar con una organización administrativa que permita el trabajo en equipo y la cohesión entre los participantes del proyecto institucional. Organizar el espacio y el tiempo que los estudiantes que permanecen en la institución, operacionalizando y haciendo uso efectivo del tiempo.

5.- Colaboración escuela - familia: Este es uno de los aspectos más importantes, ya que de ello depende el éxito del proyecto educativo y el involucramiento de las familias en el trabajo de la escuela. Los docentes deben llevar a cabo reuniones de tipo formal e informal que les permita a los padres de familia, sentirse parte del proyecto educativo en el cual participan sus hijos e hijas.

6.- Transformación de los recursos y servicios destinados a la educación especial: Este aspecto se considera básico, ya que los docentes de apoyo deben convertirse en la clave del trabajo colaborativo, destinados a participar en todo el centro, teniendo en cuenta que deben estar centrados en el currículo, y que además, es importante que en el centro participe un equipo interdisciplinario que se involucre en todo el proyecto del centro. Ramón-Laca (1998) comenta al respecto:

Parece contradictorio que hablemos de necesidades individuales y a la vez de un currículo único, de objetivos generales de grupo. Sin embargo no lo es, se trata de incorporar a los alumnos al proyecto global para su integración en la Sociedad, por eso hablamos de "escuela inclusiva" (p. 11).

También, con respecto al docente de apoyo, la autora escribe la siguiente reflexión: 
El verdadero especialista en nuestro sistema es el profesor de apoyo que suele ser un profesor de educación especial. Su misión es prestar atención individualizada a los alumnos con necesidades educativas especiales, pero por encima de esto, es el principal y más firme puntual básico de que se sirve el profesor - tutor en la difícil tarea de que se ha venido hablando hasta aquí. Con él planifica toda la actividad del aula. Al mismo tiempo, gracias a su preparación específica es el profesional que detecta y evalúa las necesidades educativas especiales de forma individualizada y proporciona las técnicas y recursos para hacerles frente (pp. 14)

Como se puede apreciar de la lectura de los párrafos anteriores, la inclusión o educación para todos es una tarea que le compete a la sociedad, pero sobre todo, a los centros educativos, los cuales deben, de alguna manera, cuestionarse sobre su proyecto de centro y su compromiso como institución para responder a las demandas de los estudiantes con necesidades educativas de su comunidad. La tarea es difícil, pero si los centros educativos pretenden brindar el apoyo para el cual han sido constituidos y cumplir con su objetivo, será necesario, abandonar las ideas de las dificultades que se presentan y avenirse a un estudio sistemático y consciente de su realidad, de tal manera que los estudiantes que conforman la comunidad institucional se vean beneficiados al participar en un proceso educativo en igualdad y equiparación de oportunidades.

Con respecto a estas ideas, es importante señalar como anota Ramón-Laca (1998)

En la atención a la diversidad se ha pasado de centrar los problemas en las limitaciones de algunos alumnos a considerar las carencias de la escuela, los fallos del sistema escolar. También se ha prestado atención a los procesos de aprendizaje, se ha aprendido a conceder importancia al cómo aprenden y maduran los niños y adolescentes, tratando de conocer los procesos mentales y circunstancias sociales y familiares que tanto condicionan la educación (p. 14).

\section{A manera de conclusión}

Los procesos de inclusión no corresponden únicamente a la Educación, sino que van más allá. Por lo tanto, es un fenómeno social y comunal que debe partir de las experiencias que se desarrollan en las interacciones de todas las personas que conviven en un contexto.

Estos procesos deben generarse de un análisis de las experiencias que se llevan a cabo desde la Educación Regular y la puesta en práctica de un currículo para todos; es desde aquí donde debe comprenderse la inclusión, y no necesariamente desde la 
Educación Especial, ya que se habla de educación para todos, sin importar cuestiones de etnias, religión, sexo ni condiciones personales o de grupo.

La inclusión no implica solamente atender a todos los estudiantes en el aula; se trata más bien de comprenderlos, escucharlos y responder a sus necesidades, intereses, características y potencialidades, sin involucrarse en actos de discriminación hacia ninguno de los participantes en el aula.

Por lo tanto, la inclusión debe partir de un análisis real de los procesos educativos que se desarrollan en cada centro educativo, o sea, debe darse un proceso contextualizado de carácter colectivo, en el cual se involucren los padres de familia, los docentes, los directores, los estudiantes y todos los miembros de la comunidad educativa.

De esta manera, la inclusión se convierte en una experiencia humanizante, donde todos se ven como miembros de una comunidad que comparte sus experiencias en el desarrollo de una sociedad más justa, democrática y solidaria.

La historia dirá qué fue lo que aportamos desde esta sociedad a los procesos de inclusión, y mientras tanto, es importante permitir que los procesos de integración mejoren la participación de nuestros estudiantes con necesidades educativas especiales en el sistema regular, como respuesta a una normativa que rige en la actualidad; así que, jadelante en nuestra tarea!.

\section{Referencias}

Asamblea Legislativa. (1996). Ley 7600: Igualdad de Oportunidades para las personas con Discapacidad en Costa Rica. San José, Costa Rica: FUNdación REhabilitación y DESarrollo.

Brown y Smith (1996). Normalisation. A reader for the nineties. London: Routlefge.

Echeita, Duk y Blanco (1995). Necesidades especiales en el aula. Formación docente en el ámbito de la Integración escolar. En: Boletín 36. Santiago de Chile: Publicaciones OREALC, UNESCO.

Heward, W. (1997). Niños excepcionales. Una introducción a la educación especial. 5ta. Edición. España: Prentice Hall. 
Lou, M. y López, N. (2000). Bases Psicopedagógicas de la Educación Especial. Madrid: Ediciones Pirámide.

Ortiz, M. (2000) Hacia una educación inclusiva. La educación especial ayer, hoy y mañana. En Siglo Cero. Vol. 31(1) pp. 5-11.

Ramón-Laca, Ma. L. (1998). El reto de la atención a la diversidad: El trabajo en el aula de integración educativa. Manuscrito sin publicar. México, Saltillo de Coahuila.

UNESCO. (1994). Declaración de Salamanca y Marco de Acción sobre Necesidades Educativas Especiales. Conferencia Mundial sobre necesidades educativas especiales: ACCESO y CALIDAD. Salamanca, España: UNESCO. 